and histologies were recorded. Data was recorded on an excel sheet and analysed using SPSS version 15 .

Results A total of 36 patients were included in the study. The median age was 42 years. Eleven percent $(n=4)$ were referred from general medicine, $22 \%(\mathrm{n}=8)$ from general surgery and $67 \%$ $(n=24)$ from Gastroenterology for endoscopic investigations. Median HB level was $10 \mathrm{~g} / \mathrm{dL}$. There were 4 patients on NSAIDs prior to referral.

Gastroscopy was performed in all patients and was normal in $94 \%(\mathrm{n}=34)$. The remaining $6 \%(\mathrm{n}=2)$ were diagnosed with $\mathrm{h}$ pylori gastritis $(n=1)$, and benign pyloric stenosis $(n=1)$. Duodenal (D2) biopsies were performed in 32 patients (89\%), of which 1 patient was diagnosed with coeliac disease on histology. Three of the 4 patients with no D2 biopsies had a normal coeliac blood screen. Lower GI endoscopy (colonoscopy $n=30$, flexible sigmoidoscopy $n=3)$ was performed in $33(92 \%)$, of which $85 \%(n=28 / 33)$ were macroscopically normal. Five patients had pathology encountered at endoscopy, these included adenomas $(n=3)$, ulcerative colitis $(n=1)$ and caecal carcinoma $(n=1)$.

Patients with IDA and no GI symptoms ( $\mathrm{n}=21$ ); endoscopic diagnostic yield was $5 \%(n=1)$ for gastroscopy and $11 \%(n=2 / 19)$ for colonoscopy. The diagnostic yield in symptomatic patients was $7 \%(n=1)$ for gastroscopy and $21 \%(n=3 / 14)$ for colonoscopy.

There were 11 patients with IDA and an HB level over $11 \mathrm{~g} / \mathrm{dL}$. All patients underwent gastroscopy with D2 biopsies and 10 patients underwent colonoscopy. All endoscopic investigations including $\mathrm{D} 2$ biopsies were normal.

Conclusion IDA in young males should always be investigated with bi-directional endoscopy, irrespective of symptoms. Although a small cohort, our study shows that the diagnostic yield is higher amongst patients with IDA and GI symptoms as well as IDA with an HB level below 11g/dL.

Disclosure of Interest None Declared.

\section{PTH-043 DIFFICULT BILIARY ACCESS: EARLY EXPERIENCE WITH THE NEW DOUBLE WIRE TECHNIQUE COMPARED TO CONVENTIONAL PRECUT AT ERCP}

doi:10.1136/gutjnl-2013-304907.530

1."R Rameshshanker, 'B Macfarlane, 'A King, 'A Leahy. 'Gastroenterology, Watford General Hospital, Watford, UK

Introduction Selective cannulation of the common bile duct (CBD) during endoscopic retrograde cholangiopancreatography (ERCP) can be technically challenging. The precut technique is a tried and tested method of gaining biliary access when standard cannulation with either contrast or guidewire has failed. However, it does have a recognised higher risk of causing pancreatitis and when used it is currently advised to place a prophylactic pancreatic stent. A new technique, termed double guidewire, involves leaving a wire in the pancreatic duct to provide a "roadmap" for subsequent biliary wire cannulation.

Objectives To report our initial experience for the success and complication rate of the double guidewire technique.

Methods Retrospective analysis of all patients who underwent ERCP between April 2010 to April 2012 at our institution. Observed differences were tested with the Fisher's exact test.

Results 484 ERCP procedures, all having initial cannulation with either a single guidewire or contrast. Overall successful biliary cannulation for first ever ERCP was achieved in 95\%. Attempted CBD cannulation was facilitated by double guidewire in 20 patients and precut in 19 patients. CBD cannulation was successful in 19/20 (95\%) double guidewire patients and 15/19 (79\%) precut patients $(\mathrm{p}=\mathrm{NS})$.
Post-ERCP pancreatitis occurred in all procedures were $11 / 484$ $(2.2 \%)$, double guidewire $3 / 20(15 \%)(p<0.02)$, precut $1 / 19(5.3 \%)$. Prophylactic pancreatic stents were placed in 2 double guidewire patients and no precut patients. In the double guidewire group, patients with pancreatitis stayed longer (mean 8 days) in the hospital when compared to precut group (mean 3 days).

Conclusion Deep CBD cannulation could be equally achieved with the help of either the double guidewire or the precut technique. There was a significant increased risk of pancreatitis with the double guidewire technique presumably related to inadvertent and repeated wire cannulations of the pancreatic duct. We find that the double guidewire technique is technically easy and currently use it if pancreatic duct cannulation occurs on $>3$ occasions. However, when used we now also place a prophylactic pancreatic stent at the end of the procedure.

Conflicts of interest none

Disclosure of Interest None Declared.

\section{PTH-044 THE SAFETY OF ERCP IN PATIENTS AGED 90 AND ABOVE}

doi:10.1136/gutjnl-2013-304907.531

1,"R Pandey, 'M Khan, ${ }^{1} \mathrm{~N}$ Van Someren, ${ }^{1} \mathrm{~K}$ Besherdas. 'Gastroenterology, Barnet \& Chase Farm NHS Trust, London, UK

Introduction The ageing population in the UK means that biliary diseases are becoming more common. Endoscopic Retrograde Cholangiopancreatography (ERCP) is therefore increasingly being used as a therapeutic strategy. However, the elderly population often have associated co-morbidities and poorer physical health, which would suggest that ERCP may be technically more challenging, or of higher risk in this group. We look at the safety of ERCP in patients over 90

Methods Patients aged 90 or above who underwent ERCP from October 1999 to October 2012 were studied retrospectively. The data was extracted from a single centre computer ERCP database.

Results ERCPs performed in patients aged 90 or above represented $6.5 \%$ of a total of 4017 procedures. The age range of patients was from 90 to 103 years. Therapeutic intervention was required in $246 / 262$ procedures (93.9\%). 16 specific types of intervention were performed, with the majority being sphincterotomy $(62.2 \%)$ or stent insertion $(16.7 \%)$. The procedure related mortality was $0.76 \%$ (2/262). 1 death occurred following a significant bleed post sphincterotomy, the other death resulted from a retroperitoneal perforation following duodenal dilatation. Complications occurred in $5.3 \%$ $(14 / 262)$ of procedures. The most frequently occurring complications were bleeding and cholangitis, each of which occurred in $3 / 262(1.1 \%)$ patients. Perforation occurred in $2 / 262$ patients $(0.76 \%)$. Pancreatitis did not occur in any of the patients. There were no complications related to co-morbidity.

Conclusion The number of ERCPs being performed in elderly patients is likely to increase. The procedure related mortality and morbidity is comparable with national figures. ERCP in this group is safe, practical and has a definite therapeutic benefit in a large proportion of patients.

Disclosure of Interest None Declared.

\section{PTH-045 ENDOSCOPIC SUBMUCOSAL DISSECTION FOR EARLY GASTRIC NEOPLASIA; PROSPECTIVE OUTCOMES FROM A SINGLE TERTIARY CENTRE}

doi:10.1136/gutjnl-2013-304907.532

1,2, $\mathrm{R} \mathrm{J}$ Haidry, ${ }^{3,4} \mathrm{M}$ Butt, ${ }^{4,5} \mathrm{~J}$ Louis-Auguste, ${ }^{6} \mathrm{M}$ Novelli, ${ }^{3,4} \mathrm{~L}$ Lovat, ${ }^{4} \mathrm{M}$ Banks. 'University College Hospital London (UCLH) NHS foundation Trust, Lonodn; ${ }^{2}$ National Medical laser Centre (NMLC), University College London; ${ }^{3} \mathrm{NMLC}$, London; ${ }^{4} \mathrm{UCLH}$, Iondon; ${ }^{5} \mathrm{NMLC}$, Lonodn; ${ }^{6}$ Department of Histopathology, UCLH, London, UK 